\title{
Has the time come to phase out codeine?
}

Previously published at www.cmaj.ca

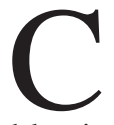
odeine is, second only to morphine, the most widely used narcotic analgesic. ${ }^{1}$ Health professionals and the public generally believe that codeine, used responsibly, is safe, a perception fostered by the availability of codeine-containing products for purchase over the counter in many countries. However, recent advances in our understanding of pharmacogenetics raise serious concerns about the safety of codeine, including emerging evidence that the narcotic can cause death even at conventional doses. Has the time come to phase out codeine altogether?

Codeine has poor analgesic properties. It is a prodrug, whose analgesic effects are almost entirely attributed to its principal metabolite, morphine. That's the problem - the pharmacokinetics of codeine are unpredictable. Because codeine has been in common use for over 200 years, it was never subjected to the regulatory requirement for the rigorous safety studies that are now mandatory for new drugs.

Many genetic factors have recently been identified that can substantially influence the rate of metabolism of codeine to morphine and, consequently, its effects. Specifically, polymorphisms occur in the cytochrome P450 isoenzyme CYP2D6 that enhance codeine metabolism to morphine. Additional polymorphisms affecting morphine metabolism, blood-brain barrier transition or opioid and opiate receptor kinetics, or combinations of these polymorphisms, may also substantially augment effects from codeine.

All of these genetic variations can have potentially serious clinical consequences. The wrong combination can result in toxic levels of morphine, even at conventional doses of codeine. For infants and young children in particular, this can be deadly, because age appears to be a key factor in susceptibility to adverse effects of morphine, possibly because of age-related maturation differences in the blood-brain barrier. Pharmacogenetic variants have been implicated in the death of a breastfed neonate whose mother had been given codeine postpartum, ${ }^{2}$ and in the death and anoxic brain injury of two young children prescribed codeine for postoperative pain following tonsillectomy for sleep apnea. ${ }^{3.4}$ Serious adverse effects from codeine due to pharmacogenetic variants, including life-threatening respiratory depression, have also been reported in adults.'

Reports of life-threatening or fatal consequences of genetic variation in codeine metabolism have been few, perhaps because testing for these gene variants is only available through research laboratories. Other evidence suggests a more widespread problem. The global distribution of the ultrafast metabolizing variant of CYP2D6, which produces high levels of morphine after codeine intake, varies by geographic origin: about $40 \%$ in North Africa, $26 \%$ in Oceania, $12 \%$ in the Middle East, $8 \%$ in North America and 3\% in Europe. ${ }^{1}$ In a recent study of opioid deaths in Ontario between 1991 and 2004, ${ }^{5}$ codeine was the only opioid used in $20 \%$ of patients, which suggests that, although some deaths may have been caused by intentional overdose, pharmacogenetic variants may have been implicated.

Because the need for oral pain control is so pervasive, the potential risk associated with codeine must be mitigated. Clearly, pretesting all patients for genetic variants before recommending codeine would be expensive and impractical. A more logical solution might be to restrict access to codeine for infants and young children, the apparent highest risk group. For breastfeeding mothers, guidelines for using codeine highlight signs and symptoms of toxic morphine levels in infants but do not call for avoiding codeine altogether. ${ }^{6}$ Health Canada is considering changing the labelling for codeine to help clarify risk. However, it is unclear at what age risk diminishes, and recommendations vary internationally: Switzerland sets the minimum age for codeine-based treatment at 10 years, the Netherlands at 1 year, the United States at 3 years and Canada at 2 years.

These approaches will likely be inadequate to address the risk posed by codeine. Perhaps a more direct approach is now needed: to stop using the prodrug codeine altogether and instead use its active metabolite, morphine. Not only is the metabolism of morphine more predictable than that of codeine, but also it's cheaper. At least one leading pediatric hospital - Toronto's Hospital for Sick Children - has already removed codeine from its formulary. Others would do well to follow its lead.

Further research, in both adults and children, should be mandated to better understand the safety of codeine. Until such research is done, professional associations and health care institutions should warn physicians of the potential for serious harm by modifying guidelines on pain control, especially for newborns and children.

\section{Noni MacDonald MD MSc \\ Section Editor, Public Health, CMAJ \\ Stuart M. MacLeod MD PhD \\ Professor of Pediatrics, University of British Columbia, \\ Vancouver, BC \\ With the editorial advisory team: Paul C. Hébert MD MHSc, Matthew B. Stanbrook MD PhD and \\ Ken Flegel MDCM MSc}

Competing interests: See www.cmaj.ca/misc/edboard.shtml. Dr. MacLeod is a consultant to the WHO Better Medicines for Children Program and a coinvestigator for the Canadian Pharmacogenomic Network for Drug Safety.

\section{CMAJ 2010. DOI:10.1503/cmaj.101411}

\section{REFERENCES}

1. Madadi P, Koren G. Pharmacogenetic insights into codeine analgesia: implications to pediatric codeine use. Pharmacogenomics 2008;9:1267-84

2. Madadi P, Ross CJ, Hayden MR, et al. Pharmacogenetics of neonatal opioid toxicity following maternal use of codeine during breastfeeding: a case-control study. Clin Pharmacol Ther 2009;85:31-5.

3. Voronov P, Przybylo HJ, Jagannathan N. Apnea in a child after oral codeine: a genetic variant — an ultrarapid metabolizer. Paediatr Anaesth 2007;17:684-7.

4. Ciszkowski C, Madadi P, Phillips MS, et al. Codeine, ultrarapid-metabolism genotype, and postoperative death. $N$ Engl J Med 2009;361:827-8.

5. Dhalla IA, Mamdani MM, Sivilotti MLA, et al. Prescribing of opioid analgesics and related mortality before and after the introduction of long-acting oxycodone. CMAJ 2009;181:891-6.

6. Madadi P, Moretti M, Djokanovic N, et al. Guidelines for maternal codeine use during breast feeding. Can Fam Physician 2009;55:1077-8. 